\title{
Non-Irritating Iodized Ethyl Esters of H. Wightiana.
}

\author{
Howard I. Cole.
}

DRING recent visits to various leprosariums, the writer was surprised to learn how many of these places had entirely given up the administration of chaulmoogra-group ethyl esters in favour of the oil because the esters had proved " too irritating and tended to produce ulcers." It is true that some years ago we also experienced trouble due to the irritating effect of the esters, but since then, we have been constantly improving this drug until we now manufacture a standardised product which never produces ulcers and which gives a very low complaint rate.

Iodized esters are to be preferred to the oil because they 
are more effective, and more rapid in their action. Some places utilise a mixture of half and half esters and olive oil, with or without the addition of 4 per cent. creosote. Although this cuts down the irritation somewhat, it also necessarily reduces the effectiveness of the drug by onehalf. This may be one reason why the esters have fallen into disrepute, but probably a more cogent reason is that there has been no general cognizance of the fact that the addition of iodine when properly made, greatly reduces the irritating effects of the esters. It is true that until recently, the methods of procedure found in the literature have given rather vague directions for this addition of iodine. Some laboratories have added the iodine to cold esters, and dissolved by stirring; others have heated the two together for varying lengths of time, and at varying temperatures, others still have heated the two together until a brown colour was produced. We have experimentally proved that both the temperature and the length of time of heating of the iodine with the esters, have an important influence on the irritating properties of the product. We have tested this time-temperature effect on over fifteen hundred cases, and find that too short, as well as too long a time of heating of the esters with the iodine increases markedly the irritant properties; there is a happy medium which we now follow with very gratifying results.

Over a period of years, we have also noted in thousands of cases the following causes of irritation :-

(1) Heating the esters to too high a temperature.

(2) Addition of less than one-half per cent, iodine.

(3) Presence of more than 0.2 per cent. free fatty acid.

(4) Presence of impurities.

It may be any one, or a combination of two or more of the above factors which is also partly responsible for the present disrepute of the esters. Whatever the cause, it no longer applies, for it is now perfectly feasible for any chemist to make non-irritating iodized esters if he will but follow our method. We now manufacture and use over 1,000 litres of iodized wightiana ethyl esters annually in our treatment clinics with no untoward results. Furthermore, our present method of intradermal injection would not be possible with an irritating product.

Our results with this drug surpass any other tried so far. Injection of about 8 c.c. in a number of small injections into a lesion, lead generally to remarkable reduction in the 
lesion, in much shorter time than has hitherto been possible with any other leprosy drug. before.

It is to be hoped that the following method of preparation of iodized ethyl esters will be tried out in other places, and that the same very encouraging results will be obtained. Complete directions for the manufacture of these esters have been described elsewhere.*

Iodized esters of low irritability, in any amount from 1 litre to 15 litres, may be prepared if the precautions given are carefully heeded.

The clear esters are placed in a shallow (pan) type of container, heated quickly to $140^{\circ} \mathrm{C}$. with occasional stirring. The requisite amount of re-sublimed iodine to give a 1.5 per cent. iodine solution in the esters is added with stirring. The temperature immediately rises to $150^{\circ} \mathrm{C}$. at which point, it is maintained for exactly 30 minutes, stirring the mixture frequently. After cooling, the iodized esters are filtered into bottles, sterilized, and then hermetically sealed.

\section{Precautions.}

The ethyl esters must be thoroughly dried before the iodine is added as otherwise an irritant product will result, since the iodine will change some of the esters to free fatty acid if water is present. If the filtered esters are clear when placed in the pan, the heating to $140^{\circ} \mathrm{C}$. before adding the iodine will drive off all dissolved water.

A shallow type of container must be used and the heating to $140^{\circ} \mathrm{C}$. done as quickly as possible; otherwise the product may be irritating. Enamel, glass or stainless steel pans may be used.

The iodised esters will keep in a dark place for at least two years without deterioration if in hermetically sealed bottles.

Iodized esters exposed to heat or sunlight in the presence of air are soon changed into a product with greatly increased irritant properties, hence the need for air-tight containers. The change is generally accompanied by an increase in clarity and a change in colour from greenish brown to red brown.

Repeated re-sterilization should be avoided as it also may lead to increase in irritant properties.

* Perkins, G. A. - Journ. Ind. Eng. Chem. 19, (1927) 939.

Cole, H. I. - Phil. Journ. Science, 40, (1929) 503. 
During the heating the esters will give off some vapour (smoke) and a slight amount of the iodine will also be volatilised by the heat, but neither of these phenomena will cause any harm to the finished product.

In order to be certain that the reaction between the iodine and the esters has gone to completion, it is wise to store the sealed bottles of iodized esters for two weeks before using. 